\title{
RESEARCH ON INDUCTION HEATING - A REVIEW
}

\author{
Vimal R. Nakum \\ Noble Group of Institution \\ Bamangam, Junagadh,
}

India

\author{
Kevin M. Vyas \\ Noble Group of Institution \\ Bamangam, Junagadh \\ India
}

\author{
Niraj C. Mehta \\ Noble Group of Institution \\ Bamangam, junagadh \\ India
}

\begin{abstract}
This paper presents results of finite element analysis of induction heating problems considering temperature dependence of material characteristics. In this analysis, we have used the three-dimensional finite element method in order to correctly express induction heating coil's shapes and to make clear its effects on temperature distributions. The heat-conducting problem and the eddy current problem are coupled, and solved by using the step-by-step calculations.
\end{abstract}

Keywords: Magnetic field, Open-type magnetizer, Toroidal

\section{INTRODUCTION}

In the conventional finite element analysis for linear induction heating problems, magnetic material parameters are usually assumed to be constants with respect to temperature. As for the non-linear analysis, the temperature dependence of the electrical conductivity is easily taken into consideration; however, the temperature dependence of the magnetic permeability is usually neglected due to its difficulty because there are no catalog data concerned on temperature dependence of the magnetic permeability and such properties are very difficult to measure [1-3].

In the designing of induction heating devices, we should measure and consider the dependence of the magnetic permeability to obtain more accurate solutions. Generally the permeability is measured under a constant magnetic field condition with toroidal cores because the magnetic field strength directly proportional to the exciting current. Disadvantage of this measuring method is that it takes long time in making toroidal cores and those windings.

On the other hand, the magnetic flux density is directly obtained from the magnetic vector potential distributions in the case of the finite element analysis. The magnetic field strength is obtained finally from the magnetic flux density value and the magnetic permeability value in each element with the constitutive relation.

Therefore, it is very convenient to use the relationship between the magnetic flux density, the magnetic permeability and temperature as a function in the numerical computations. In this paper, such functions of the magnetic permeability at any temperature under constant flux density conditions are derived from the measured data. The numerical analysis considering the temperature dependence of the permeability give us more important knowledge in designing modern induction heating devices.

\section{MATERIAL CHARACTERISTICS[4]}

Conventionally, we have measured the temperature dependence of the magnetic permeability by using toroidal cores and platinum exciting coils and pickup coils as shown in Fig. 1, in order to realize a high temperature condition. The platinum wires are used to prevent melting metal.

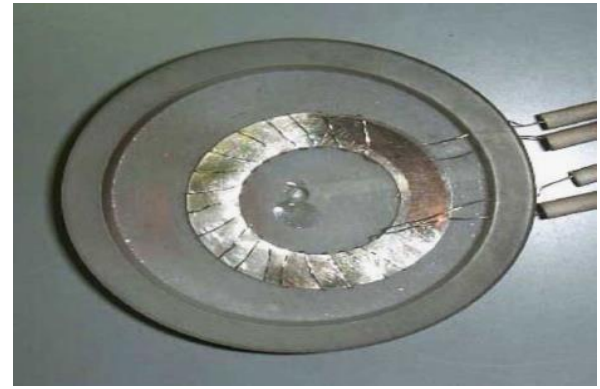

Fig. 1 ring sample

However, it needs a long preparing time to change specimen in a ring-type core because it is necessary to renew the windings and reconnect the platinum wires with the terminals for taking out the pickup signals from the vacuum chamber [2-3]. Fig. 2 shows a newly developed open-type magnetizer. The magnetic properties of a ribbon specimen can be measured by only inserting it through the quartz holder, which has the fixed exciting coil and the pickup coil.

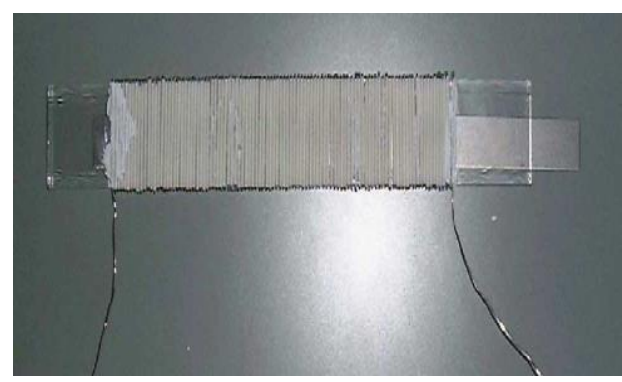

Fig. 2 Magnetizer for a plate sample

The numbers of windings of the exciting coil and the pickup coil are 150 turns and 16 turns, respectively. The alumina pipes were used in insulation of the windings. In the verification of this measuring apparatus, a SS400 sample was measured. In the measurement, the exciting frequency was changed from $100 \mathrm{~Hz}$ to $100 \mathrm{kHz}$ with a $\mathrm{B}-\mathrm{H}$ analyzer, and the field strength was also changed to be from $200 \mathrm{~A} / \mathrm{m}$ to $1600 \mathrm{~A} / \mathrm{m}$. Figs. 3 and 4 show the measured hysteresis loci when the exciting frequency is $100 \mathrm{~Hz}$ and $100 \mathrm{kHz}$, 
respectively. In these figures, the loop measured with the new open-type magnetizer was compared with the conventional one. There were good agreements between the results obtained with each magnetizer in high-frequency exciting condition (see Fig. 4) [4].
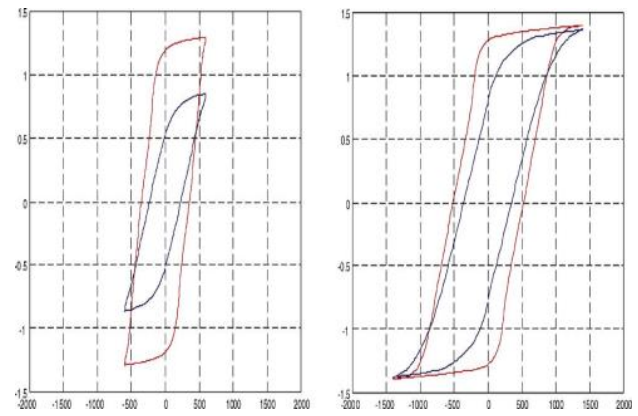

Fig. 3 the measured data of B-H curve at $100 \mathrm{~Hz}(\mathrm{H}-$ constant)
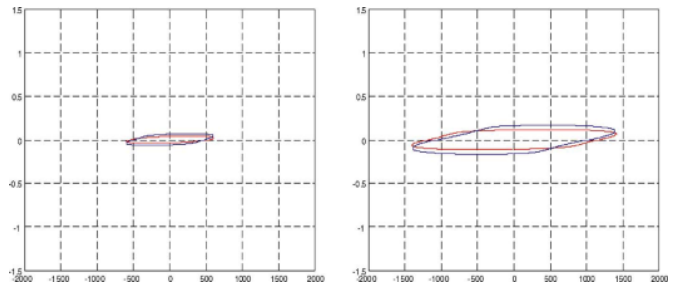

Fig. 4 the measured data of $\mathrm{B}-\mathrm{H}$ curve at $100 \mathrm{kHz}(\mathrm{H}-$ constant)

It can be said that the simplified open-type magnetizer is useful in measuring many sample sheets under high frequency conditions during a short term of measurement. Since the purpose of this measurement is to obtain the data depending on temperature, which is applied to the non-linear magnetic field and thermal conducting analysis. Fig. 5 shows the measurement system. As shown in this figure, the specimen placed in the quartz vacuum chamber is being heated by the infrared ray heating device. The temperature of specimen was measured with a platinum-rhodium thermo couple and the temperature was controlled with the personal computer (PC) and the program controller. The $\mathrm{B}-\mathrm{H}$ analyzer was used for the measurement of the magnetic properties. The specimen used in this measurement was SUS430. In the measurements, the exciting frequency was changed from $100 \mathrm{~Hz}$ to $500 \mathrm{kHz}$, and the magnetic field intensity was changed from $50 \mathrm{~A} / \mathrm{m}$ to 800 A/m. Figs. 6 and 7 show the measured results for $100 \mathrm{kHz}$ and $100 \mathrm{kHz}$, respectively. As shown in these figures, the magnetic permeability can be expressed as a function of the temperature and the magnetic flux density (a) (ortho magnetic field strength (b)).

The permeability changed quickly around the Curie point. Because the large exciting power needed to input in highfrequency measurements, it was difficult to obtain the data in the saturation region of the magnetization. To obtain such data at a high-frequency used in numerical computations, we used an approximation with the following function [5].

$\sum_{i=0}^{n}\left[\begin{array}{l}n \\ i\end{array}\right] p_{i} \cdot t^{i}(1-t)^{n-i}$

Where, $(n, i)$ is the combine parameter, which can be derived as a coefficient when $(a+b) n$ is expanded in progression.

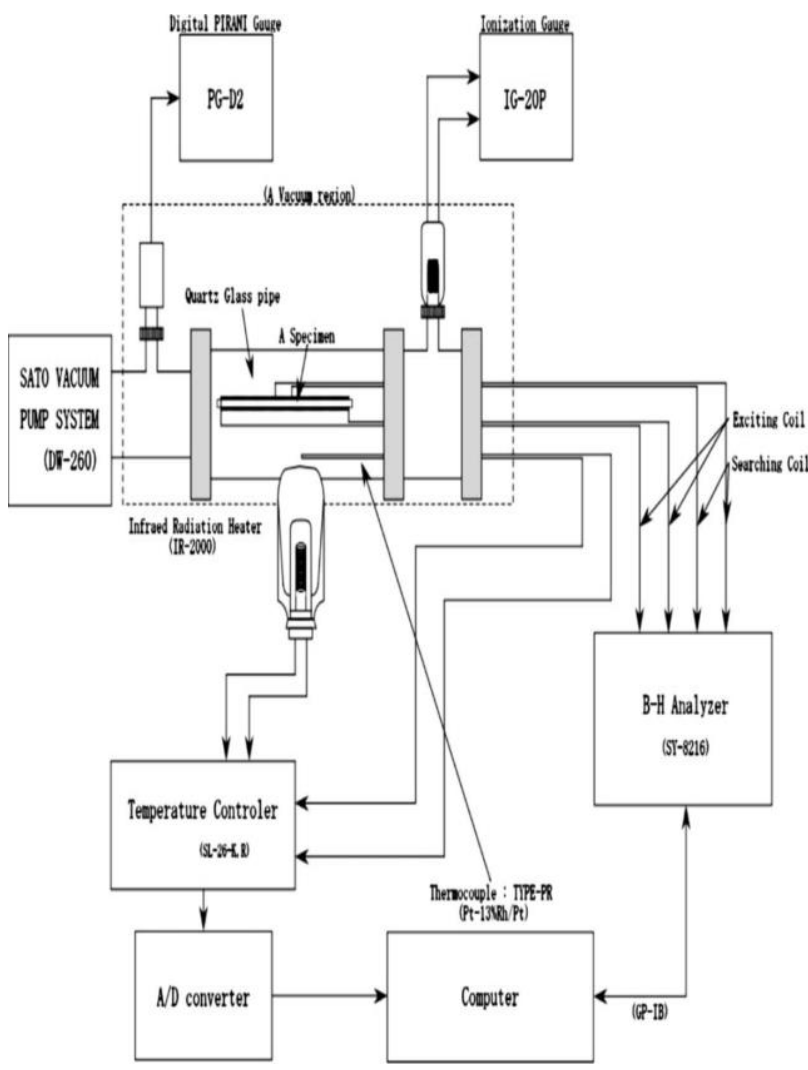

Fig. 5 The measurement system for the property depending on temperature (a) B-constant and (b) H-constant

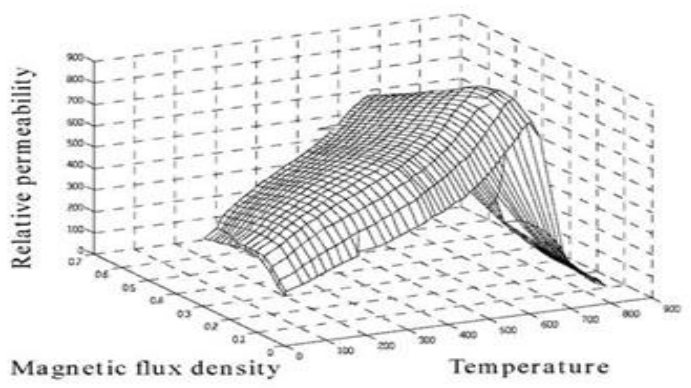

(a) 


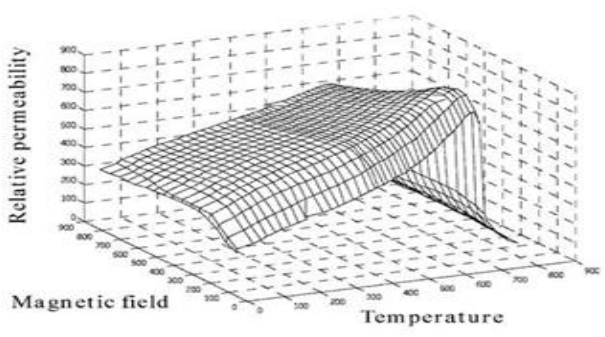

(b)

Fig. 6 Three-dimensional distribution of relative permeability at $10 \mathrm{kHz}$ (a) B-constant and (b) H-constant

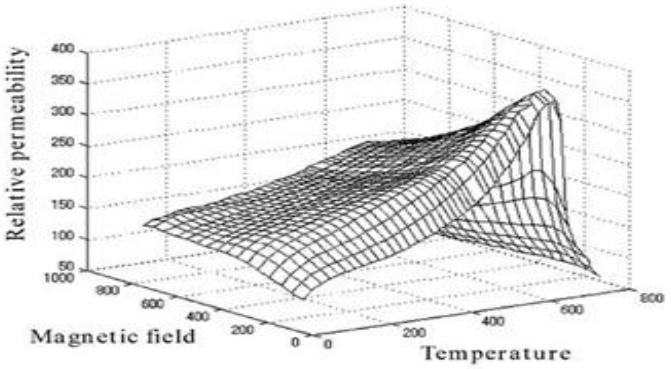

(a)

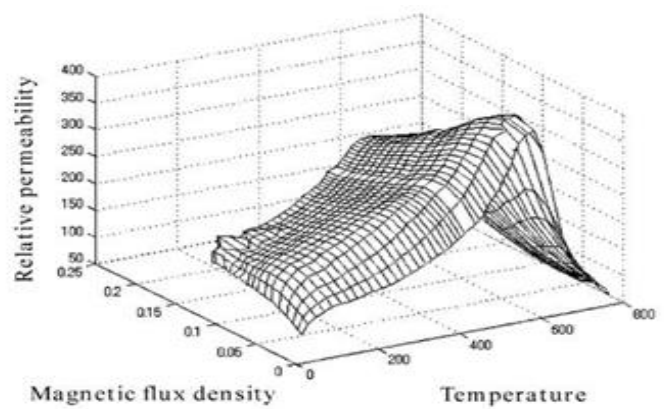

(b)

Fig. 7 Three-dimensional distribution of relative permeabilityat $100 \mathrm{kHz}$. (a) B-constant and (b) H-constant

\section{FORMULATION[9]:}

The three-dimensional finite element equation with the edge element is given as follows.

$$
\begin{aligned}
G_{i}= & \int_{V} \operatorname{rot} N_{i} \cdot(v \operatorname{rot} A) \mathrm{d} V-\int_{V} N_{i} \cdot J_{0} \mathrm{~d} V \\
& +\int_{V} N_{i} \cdot \sigma\left(\frac{\partial A}{\partial t}+\operatorname{grad} \phi\right) \mathrm{d} V=0
\end{aligned}
$$

Where, $\mathrm{A}$ is the magnetic vector potential, $\mathrm{J}_{0}$ the exciting current density, $\sigma$ the electrical conductivity and $\mathrm{N}_{\mathrm{i}}$ is the vector interpolation function of the tetrahedral element. $\mathrm{N}_{\mathrm{i}}$ can be written as,

$$
N_{i}=\lambda_{\text {me }} \operatorname{grad} \lambda_{\text {ne }}-\lambda_{\text {ne }} \operatorname{grad} \lambda_{\text {me }}
$$

Where, $\lambda$ is the volume coordinate at node. The governing equation of the transient heat problem is written as,

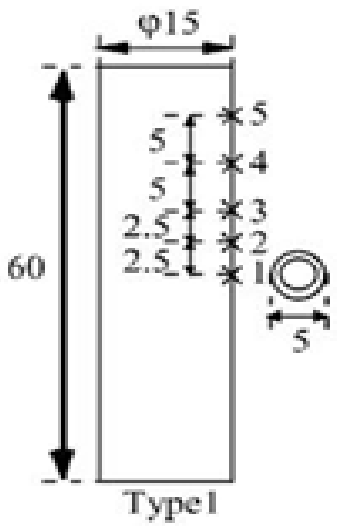

Fig. 8 Structure of model (Type-1)

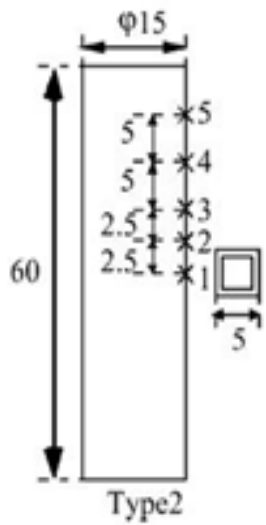

Fig. 9 Structure of model (Type-2)

$\rho c \frac{\partial T}{\partial t}=\frac{\partial}{\partial x}\left(\lambda_{x} \frac{\partial T}{\partial t}\right)+\frac{\partial}{\partial y}\left(\lambda_{y} \frac{\partial T}{\partial t}\right)+\frac{\partial}{\partial z}\left(\lambda_{z} \frac{\partial T}{\partial t}\right)+Q$

Where, $\rho$ is the density, $c$ the specific heat and $Q$ is the quantity of heat.

\section{RESULTS AND DISCUSSIONS[6-8]:}

The three-dimensional finite element analysis was carried out by taking into account of the temperature dependence of the material properties. Figs. 8 and 9 show the models used in the analysis and measurement. We have used three models; however we show here two typical cases. 
The cross-points indicated on the conductor surface are measured points with thermo couple. The Type- 1 has a circular cross section of the induction coil and Type- 2 has a square cross section. Figs. 10 and 11 show the calculated flux distributions and the comparison of the calculated temperature on the conductor surface with the measured ones. There was a good agreement between the measured and calculated results. It was evident that the flux did not go through inside of the heating conductor due to the skin effect.
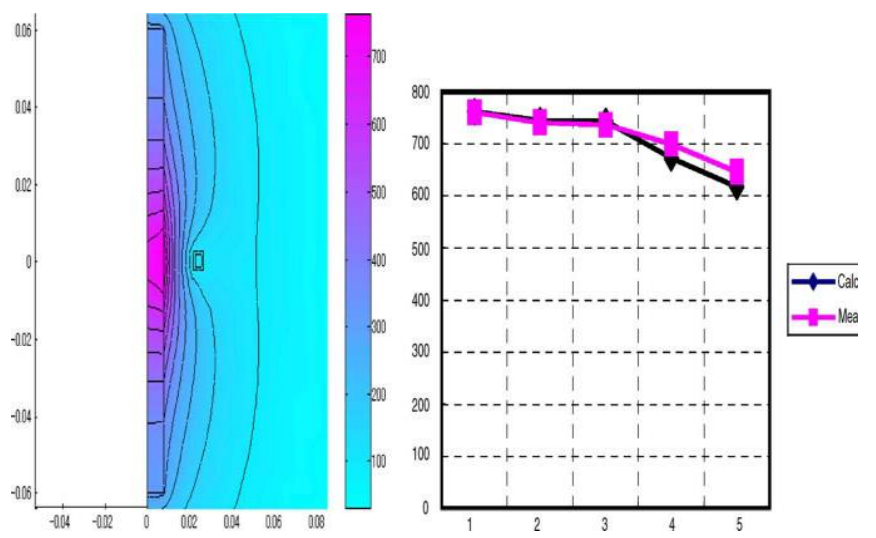

Fig. 10 Temperature distribution for Type-1
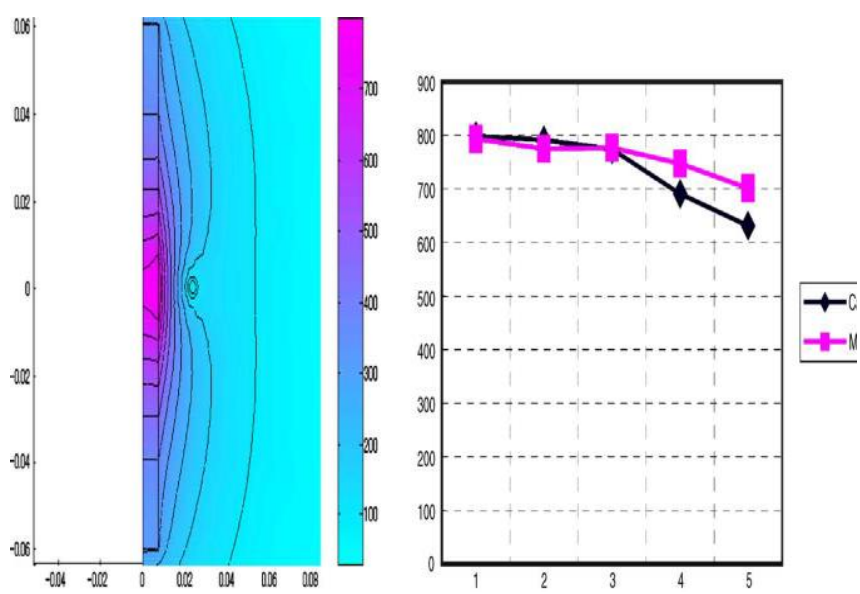

Fig. 11 Temperature distribution for Type-2

\section{CONCLUSION:}

The results obtained in this work can be summarized as follows [10]:

(1) In this paper, the new simplified open-type magnetizer has been presented in order to reduce measuring time. The measurement by using the new magnetizer can be performed by only changing a ribbon sample inside. Accurate measured results similar to the toroidal core type were able to be obtained under high-frequency conditions.

(2) In the measurements, the magnetic permeability in material has been measured as a function of the temperature and the exciting frequency (including eddy
Current effect). Those characteristics were made clear for the SUS340 specimen.

(3) In the verification, the three-dimensional finite element method was applied to the simple induction heating models and the calculated results were compared with the measured ones. The measured data mentioned in the above was used in the measurement and we obtained the good agreement between the measured and calculated results.

\section{REFERENCES}

[1] S. Midson, K. Brissing, Semi-solid casting of aluminum alloys: a status report, Mod. Cast. (1997) 41-43.

[2] S. Midson,V. Rudnev, R. Gallik, Semi-solid processing of aluminum alloys, Ind. Heat. (1999) 37-41.

[3] H.K. Jung, C.G. Kang, Induction heating process of an Al-Si alum minum alloy for semi-solid die casting and its resulting microstructure, J. Mater. Process. Technol. 120 (2002) 355-364.

[4] Y. Ono, C.Q. Zheng, F.G. Hamel, R. Charron, C.A. Loong, Experimental investigation on monitoring and control of induction heating process for semi-solid alloys using the heating coil as sensor, Meas. Sci. Technol. 13(2002) 1359-1365.

[5] D.-C. Ko, G.-S. Min, B.-M. Kim, J.-C. Choi, Finite element analysis for the semi-solid state forming of aluminum alloy considering induction heating, J. Mater. Process. Technol. 100 (2000) 95-104.

[6] M.C. Flemings, Behavior of metal alloys in the semisolid state, Metall. Trans. A 22 (1991) 957-981.

[7] K.T. Nguyen, A. Bendada, An inverse approach for the prediction of the temperature evolution during induction heating of a semi-solid casting billet, Modell. Simul. Mater. Sci. Eng. 8 (2000) 857-870.

[8] S. Midson, V. Rudnev, R. Gallik, The induction heating of semi-solid aluminum alloys, in: Proceedings of the Fifth International Conference on Semi-Solid Processing of Alloys and Composites, Golden, CO, 1998, pp. $497-$ 504.

[9] A. Bendada, K.T. Nguyen, C.A. Loong, Application of infrared imaging in optimizing the induction heating of semi-solid aluminum alloys, in: Proceedinmgs of the International Symposium on Advanced Sensors for Metals Processing, Quebec, 1999, pp. 331-342.

[10] P. Kapranos, R.C. Gibson, D.H. Kirkwood, C.M. Sellars, Induction heating and partial melting of high melting point thixoformable alloys, in: Proceedings of the Fourth International Conference on Semi-Solid Processing of Alloys and Composites, Sheffield, 1996, pp. 148-152. 